\title{
Black Phosphorus-Film With Drop-Casting Method for High-Energy Pulse Generation From Q-Switched Er-Doped Fiber Laser
}

\author{
Huanhuan LIU, Wei SONG, Ye YU, Qiao JIANG, Fufei PANG*, and Tingyun WANG \\ Key Laboratory of Specialty Fiber Optics and Optical Access Networks, Joint International Research Laboratory of \\ Specialty Fiber Optics and Advanced Communication, Shanghai Institute for Advanced Communication and Data \\ Science, Shanghai University, Shanghai 200444, China \\ *Corresponding author: Fufei PANGＥ-mail: ffpang@shu.edu.cn
}

\begin{abstract}
We have reported high-energy pulse generation from a Q-switched Er-doped fiber laser based on black phosphorus (BP) with a drop-casting method. Poly dimethyl diallyl ammonium (PDDA) is employed to protect BP. A passive Q-switching operation is achieved by using the $\mathrm{BP} /$ polyvinyl alcohol (PVA) film as the saturable absorbers (SAs). When the pump power increases from $130.4 \mathrm{~mW}$ to $378 \mathrm{~mW}$, the repetition rate increases from $13.33 \mathrm{kHz}$ to $26.6 \mathrm{kHz}$, and the pulse duration decreases from $10.67 \mu$ s to $7.11 \mu \mathrm{s}$. The maximum pulse energy is $468.03 \mathrm{~nJ}$, to the best of our knowledge, which is the highest pulse energy produced from Q-switched fiber laser based on BP-SA at $1.5 \mu \mathrm{m}$. The obtained high-energy pulse demonstrates that BP/PVA film fabricated by such a drop-casting method can provide an ideal SA for high pulse energy generation from fiber lasers, and it has a potential application of remote sensing.
\end{abstract}

Keywords: Fiber laser; Q-switched; black phosphorus.

Citation: Huanhuan LIU, Wei SONG, Ye YU, Qiao JIANG, Fufei PANG, and Tingyun WANG, "Black Phosphorus-Film With Drop-Casting Method for High-Energy Pulse Generation From Q-Switched Er-Doped Fiber Laser," Photonic Sensors, 2019, 9(3): 239-245.

\section{Introduction}

Q-switched fiber lasers with high pulse energy have attractive applications in medicine and remote sensing including light detection and ranging, optical time-domain reflectometry, and gas detection [1]. Compared with the active Q-switching, passive Q-switching possesses the advantages of simple and compact structure [2]. The passive Q-switched fiber laser is achieved by incorporating with saturable absorbers (SAs). Due to quantum confinement and surfaces, which are naturally passivated without any dangling bonds [3], recently, the up-coming two-dimensional (2D) materials have a large potential to be ideal SAs in fiber lasers with merits of broadband absorption, and wavelengthindependent saturable absorption including graphene, transition metal dichalcogenides (TMDCs), and topological insulator (TI). Graphene can assist pulsed fiber laser to operate in the near-infrared region $(1 \mu \mathrm{m}-2 \mu \mathrm{m})$ [4]. However, the low modulation depth $(\sim 1 \%)$, as a result of the relative weak absorption in graphene $(\sim 2.3 \%$ per layer $)$, would limit its light modulation ability [5]. Lately, TMDCs have been applied in mode-locked fiber laser because of their higher resonant absorption at

Received: 29 November 2018 / Revised: 13 March 2019

(C) The Author(s) 2019. This article is published with open access at Springerlink.com

DOI: $10.1007 / \mathrm{s} 13320-019-0549-6$

Article type: Regular 
special wavelengths [6]. Nevertheless, their large bandgap ( $1 \mathrm{eV}$ for bulk and $2 \mathrm{eV}$ for monolayer) limits their application in telecommunication band [7]. The topological insulator (TI) as another novel two-dimensional (2D) nanomaterial has proved to be an ideal SA due to its large modulation depth and high damage threshold [8]. However, the complex preparation due to compound with two different elements limits its application in pulsed laser [9].

Black phosphorus (BP), a newly emerged 2D material, has been brought into the fold as an attractive alternative to other $2 \mathrm{D}$ materials in infrared photonics and optoelectronics due to its tunable direct bandgap from $0.3 \mathrm{eV}$ to $2 \mathrm{eV}$ by changing the layer number [10]. Therefore, BP is suitable for fabricating photonic devices operating at optical communication band $(0.8 \mathrm{eV})$ and fills up the gap between the graphene and TMDCs [11]. In terms of optical properties, many experimental researches about BP have been reported. Wang et al. revealed that BP has a faster carrier relaxation in near- and mid-infrared region than graphene [12]. Ryan et al. studied the carrier dynamics in BP by using pump-probe transmission and refection measurements [13]. Zhang et al. proved that relatively large ground-state transition probability and weak loss of excited-state absorption result in the good saturable absorption performance of BP [14]. Zhang et al. reported that the nonlinear absorption coefficient of BP is dependent on its size [15]. Up to now, many Q-switched and mode-locked fiber laser based on BP at different wavelengths have been reported [16-21]. Liu et al. reported a Q-switched laser based on BP with pulse energy of $283.91 \mathrm{~nJ}$ [22]. This is the highest single pulse energy at $1.5 \mu \mathrm{m}$ based on BP as the Q-switcher.

In this work, we demonstrate the generation of pulse with energy of $\sim 468.03 \mathrm{~nJ}$ from a Q-switched Er-doped fiber laser (EDFL) by using a BP/polyvinyl alcohol (PVA) film as SAs. The few-layer BP is absorbed on the film by using a drop-casting method. Compared with the deposition of BP on fiber end directly, this method is easier to transfer BP and control the layer of BP. By inserting BP-film into EDFL, Q-switched laser at $1595 \mathrm{~nm}$ is obtained. The pulse is stable when the pump power increases from $130.4 \mathrm{~mW}$ to $378 \mathrm{~mW}$. Meanwhile, the pulse duration decreases from $10.67 \mu$ s to $7.11 \mu \mathrm{s}$, and the repetition rate increases from $13.33 \mathrm{kHz}$ to $26.6 \mathrm{kHz}$. The highest pulse energy of $468.03 \mathrm{~nJ}$ is achieved at the pump power of $298.7 \mathrm{~mW}$. To the best of our knowledge, $468.03 \mathrm{~nJ}$ is the highest pulse energy among Q-switched Er-doped fiber laser with $\mathrm{BP}$ as the SAs.

\section{Fabrication of PVA-BP-PVA film}

In our experiment, the few-layer BP solution is prepared by the liquid-phase exfoliation method (LPE) due to its simplicity and high efficiency [23]. Ultra-water is selected as the solvent for the BP exfoliation. The solvent n-methyl-pyrrolidone (NMP) can provide stable and highly concentrated BP dispersion [24]. However, NMP is an organic solvent with poor volatility [25]. Compared with NMP, water evaporates easily without affecting the photoelectric properties of BP. More importantly, poly dimethyl diallyl ammonium (PDDA) can protect BP after it dissolves in water [26]. Generally speaking, PDDA $(10 \mu \mathrm{L})$ is added into ultra-water $(10 \mathrm{~mL})$ and sonicated for 5 minutes. Then, BP powder $(5 \mathrm{mg})$ is exfoliated in as-prepared water $(0.5 \mathrm{mg} / \mathrm{mL})$ by using bath ultrasonication (operating at $40 \mathrm{kHz}$ ) for two hours. After the sonication step, to remove the thick and unexfoliated $\mathrm{BP}$, the mixture dispersion is centrifuged at $7000 \mathrm{rpm}$ for $10 \mathrm{~min}$. Finally, the $70 \%$ supernatant is collected for further study. The image of the as-prepared BP solution is shown in Fig. 1(a).

The nanosheets are characterized by Raman spectroscopy with a $633 \mathrm{~nm}$ laser. As depicted in Fig. 1(a), three primary Raman peaks are observed at $361.0 \mathrm{~cm}^{-1}, 437.9 \mathrm{~cm}^{-1}$, and $466.0 \mathrm{~cm}^{-1}$, which are corresponding to $A_{g}^{1}, B_{2 g}$, and $A_{g}^{2}$ vibration 
modes, respectively. The transmission electron microscope (TEM) image is obtained to show the morphologies of few-layer BP nanosheets. As shown in Fig. 1(b), BP nanosheets can be clearly identified as a layered structure with several hundred nanometers in size. In order to identify the thickness of the few-layer BP nanosheets precisely, the samples are measured by the atomic force microscopy (AFM). Figure 1(c) depicts the AFM image of BP nanosheets. The bright spot in Section $\mathrm{A}$ is due to the superposition of few-layer BP nanosheets. Figure 1(d) shows the height difference between the substrate and the BP nanosheets along the marked line. The thickness of $\mathrm{BP}$ is estimated to be $\sim 5.2 \pm 0.8 \mathrm{~nm}$. For all the prepared BP nanosheets within the AFM image, the thickness of $\mathrm{BP}$ is ranging from $3 \mathrm{~nm}$ to $8 \mathrm{~nm}$. With the thickness of single layer around $0.6 \mathrm{~nm}$ [27], the thickness of BP-nanosheets is around 5 to 13 layers, which is suitable to work at $1550 \mathrm{~nm}$.

To ensure that there is enough BP on the film, we adopt an improved drop-casting method. As shown in Fig. 2(a), the BP solution is dropped on the surface of a pure PVA film. BP nanosheets will adsorb on the surface of PVA film after the water evaporates. This step is repeated for three times to ensure that enough BP nanosheets are adsorbed on the film. Then, another pure PVA film is covered with BP. Finally, we put the sample into a packing bag. A vacuum sealing machine is used to remove the air from the packing bag and is sealed. About 4 hours later, the two PVA films will adhere tightly together. The PVA-BP-PVA film is attached to the end of a standard optical ferrule to operate as SAs. We can observe from Figs. 2(b) and 2(c) that the number of BP on film, which is fabricated by our improved drop-casting method, has been significantly increased.

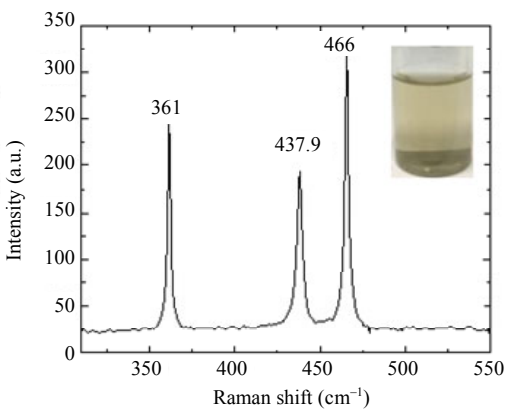

(a)

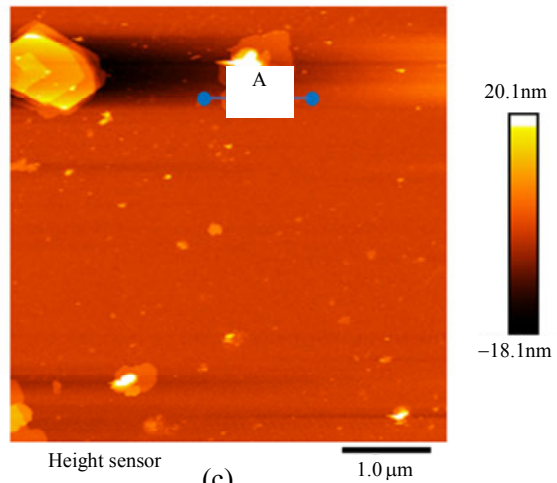

(c)

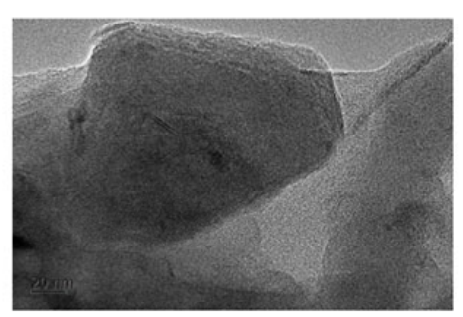

(b)

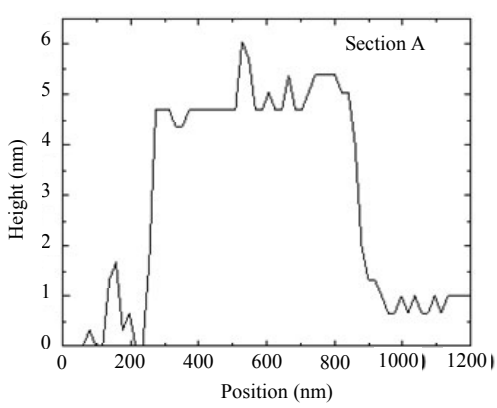

(d)

Fig. 1 Characterization of the exfoliated BP layers: (a) Raman spectrum of BP nanosheets (inset: photograph of a prepared BP solution), (b) TEM images of BP nanosheets, (c) AFM image of BP nanosheets, and (d) height profile marked in (c) (x-axis represents the position along the line and $y$-axis is the corresponding height). BP: black phosphorus; TEM: transmission electron microscope; AFM: atomic force microscopy. 


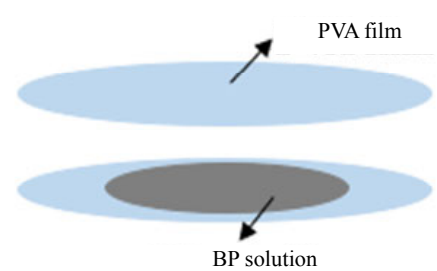

(a)

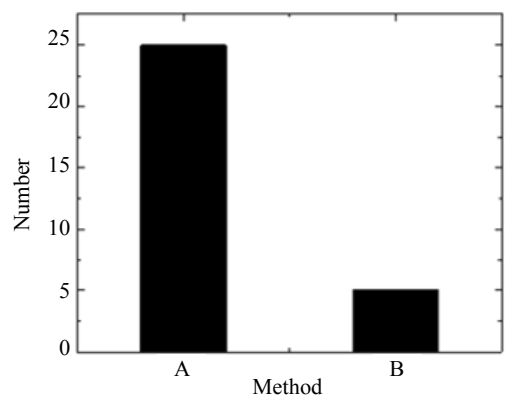

(c)
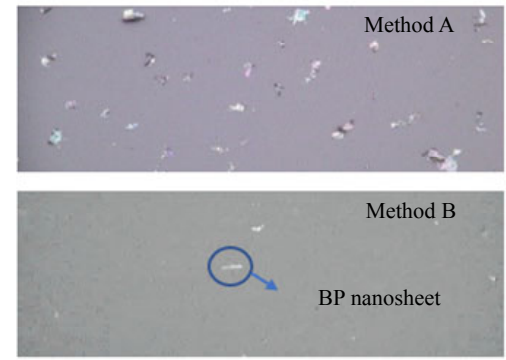

(b)

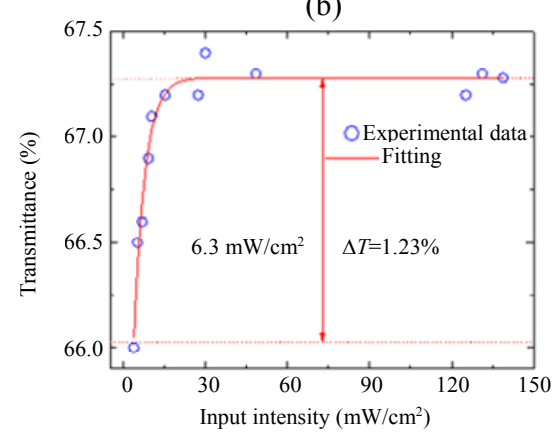

(d)

Fig. 2 Preparation of PVA-BP-PVA film and the saturable absorption measurement of BP-SA: (a) schematic diagram of the fabrication process of PVA-BP-PVA film, (b) picture of BP-film which is fabricated by improved drop-casting method (method A) and wet-chemistry techniques (method B), (c) statistics on the amount of BP nanosheets, and (d) the nonlinear saturable absorption of BP-SAs. BP: black phosphorus; PVA: polyvinyl alcohol; SA: saturable absorber.

To confirm the saturation absorption, the optical properties of the PVA-BP-PVA film can be measured by a mode-locked fiber laser [28, 29], which is operated at $1569 \mathrm{~nm}$ with $943 \mathrm{fs}$ pulse width and $60.32 \mathrm{MHz}$ repetition rate in our experiment. As shown in Fig. 2(d), the transmittance of SA increases with an increase in the input light intensity. The experimental data are well fitted by using the following formula:

$$
T(I)=1-\Delta T \times \exp \left(\frac{I}{I_{\text {sat }}}\right)-T_{\text {ns }}
$$

where $T(I)$ is the transmission, $\Delta T$ is the modulation depth, $I$ is the input intensity, $I_{\text {sat }}$ is the saturation intensity, and $T_{\mathrm{ns}}$ is the non-saturable absorbance. The modulation depth, saturation intensity, and non-saturable absorbance of SAs are $1.23 \%, 6.3 \mathrm{MW} / \mathrm{cm}^{2}$, and $32.8 \%$, respectively. The results prove that SAs based on BP can meet the essential criteria of SAs for pulsed laser.

\section{Experimental setup}

The configuration of the high compact all-fiber
EDFL is constructed to investigate the Q-switching ability of SA based on BP, as shown in Fig. 3. A piece of 7.26-m erbium-doped fiber (Er30) is employed as the gain medium. The rest fiber of the cavity consists of 1.6-m HI1060 wavelength division multiplexer (WDM) fiber and a 14.14-m-long standard single mode fiber (SMF-28). The total length of the cavity is $23 \mathrm{~m}$, and all fibers work at anomalous dispersion regime. The laser is pumped by a $980 \mathrm{~nm}$ laser diode via a $980 / 1550 \mathrm{~nm}$ WDM. A polarization-independent isolator is employed in the ring cavity to ensure the unidirectional operation of the laser. The cavity polarization state as well as the intracavity birefringence is adjusted via a polarization controller (PC). $10 \%$ of light is extracted from the laser as the output by an optical coupler. Considering that the insertion loss of BP-SA is $1.8 \mathrm{~dB}$, the cavity loss is around $3.42 \mathrm{~dB}$. The performance of pulses is analyzed by an optical analyzer (Yokogawa AQ6375) with a spectral resolution of $0.2 \mathrm{~nm}$ and a real-time oscilloscope (Tektronix MSO 4104) via a fast photodetector. 


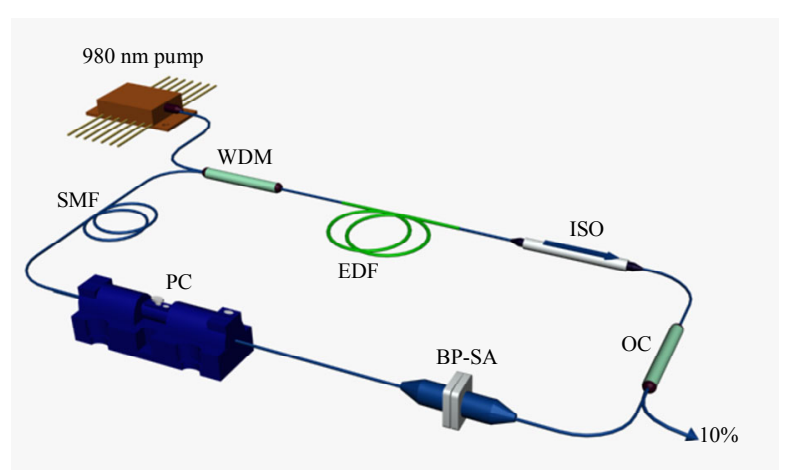

Fig. 3 Experimental setup of an erbium-doped Q-switched fiber laser based on BP-SA. WDM: wavelength division multiplexing; EDF: erbium-doped fiber; ISO: isolator; OC: output coupler; BP-SA: black-phosphorus-based saturable absorber; PC: polarization controller; SMF: single mode fiber.

\section{Results and discussion}

Before inserting the SA into the cavity, we investigate the operation of the fiber laser without SAs to see if there is any self-Q-switching effect. No matter how we change the pump power and intracavity polarization state, only continuous wave $(\mathrm{CW})$ can be observed. It clearly proves the function of SA based on BP, then the SA is inserted into the cavity.

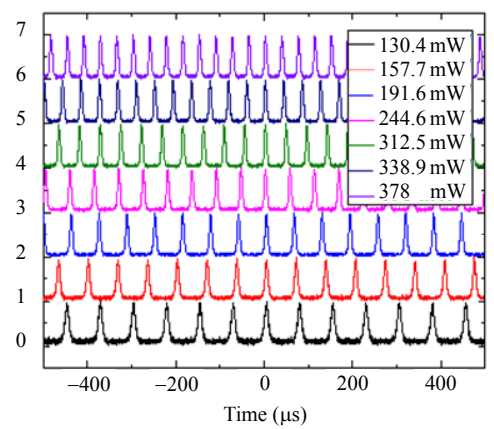

(a)

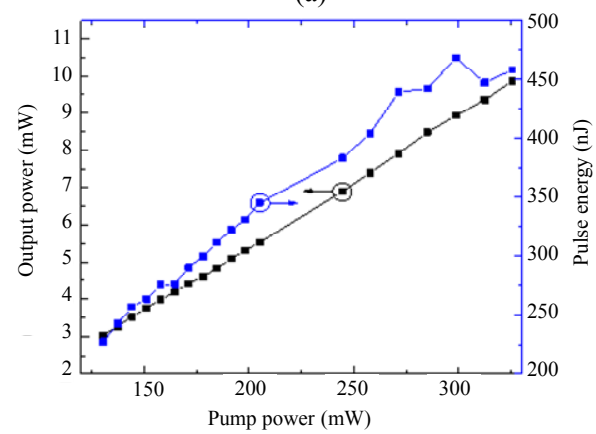

(c)
When the pump power increases to $49.1 \mathrm{~mW}$, the EDFL generates the CW. The Q-switching performance with a repetition rate of $13.33 \mathrm{kHz}$ is observed at the pump power of $130.4 \mathrm{~mW}$. The corresponding pulse energy is 227.30 nJ. Figure 4(a) shows different Q-switched pulses at the pump power of $130.4 \mathrm{~mW}, 157.7 \mathrm{~mW}, 191.6 \mathrm{~mW}$, $244.6 \mathrm{~mW}, 312.5 \mathrm{~mW}, 338.9 \mathrm{~mW}$, and $378 \mathrm{~mW}$, respectively. Figure 4(b) depicts the pulse width and repetition rate of Q-switched pulses versus pump power. The repetition rate increases from $13.33 \mathrm{kHz}$ to $26.6 \mathrm{kHz}$ with the increasing pump power from $130.4 \mathrm{~mW}$ to $378 \mathrm{~mW}$. The pulse duration goes down from $10.67 \mu$ s to $7.11 \mu \mathrm{s}$ versus the pump power. This is consistent with the characteristic of Q-switched pulses. Figure 4(c) demonstrates the average output power and pulse energy versus pump power. The average output power increases linearly from $3.03 \mathrm{~mW}$ to $9.86 \mathrm{~mW}$, and pulse energy increases from $227.3 \mathrm{~nJ}$ to $468.03 \mathrm{~nJ}$. When the pump power is $298.7 \mathrm{~mW}$, the highest pulse energy of $468.03 \mathrm{~nJ}$ is achieved.

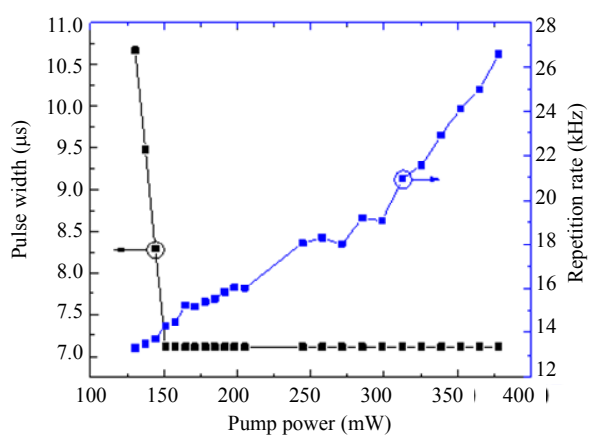

(b)

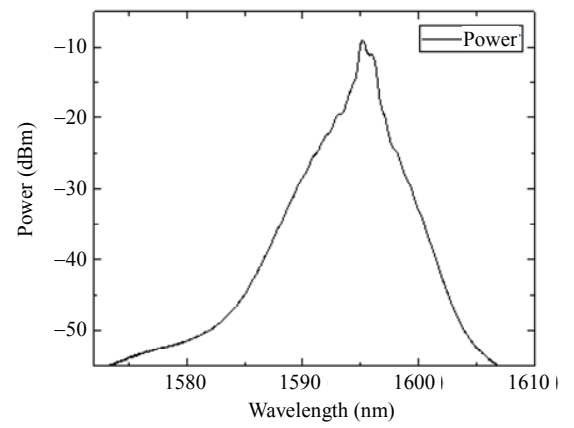

(d)

Fig.4 Performance of the developed Q-switched fiber laser: (a) different pulse trains under different pump powers, (b) pulse width and repetition rate versus pump power, (c) output power and pulse energy versus pump power, and (d) optical spectrum. 
Table 1 summarizes the Q-switched fiber laser results based on BP in recent reports. To the best of our knowledge, $468.03 \mathrm{~nJ}$ is the highest pulse energy at $1.5 \mu \mathrm{m}$ based on BP-SA. Figure 4(d) shows the spectra of the Q-switched pulses with central wavelength of $1595 \mathrm{~nm}$.

Table 1 Typical Q-switched fiber lasers based on BP.

\begin{tabular}{cccc}
\hline Repetition rate $(\mathrm{kHz})$ & Pulse width $(\mu \mathrm{s})$ & Pulse energy $(\mathrm{nJ})$ & Reference \\
\hline $7.86-34.32$ & 2.96 & 194 & {$[30]$} \\
$6.98-15.78$ & 10.32 & 94.3 & {$[18]$} \\
$35.7-70.6$ & $6.2-1.65$ & $8.7-25.2$ & {$[31]$} \\
40 & 3.1 & 18.6 & {$[32]$} \\
30.098 & 2.988 & 283.91 & {$[22]$} \\
$13.33-26.6$ & 7.11 & 468.03 & Our work \\
\hline
\end{tabular}

\section{Conclusions}

We demonstrate a Q-switched fiber laser at $1.5 \mu \mathrm{m}$ based on PVA-BP-PVA film with a drop-casting method. The modulation depth, saturation intensity, and non-saturable absorbance of BP are $1.23 \%, 6.3 \mathrm{~mW} / \mathrm{cm}^{2}$, and $32.8 \%$, respectively. By inserting the BP-film into the cavity, a Q-switched laser at $1595 \mathrm{~nm}$ is obtained. The repetition rate increases from $13.33 \mathrm{kHz}$ to $26.6 \mathrm{kHz}$ with an increase in pump power from $130.4 \mathrm{~mW}$ to $378 \mathrm{~mW}$. The shortest pulse duration is $7.11 \mu \mathrm{s}$, and the maximum pulse energy is $468.03 \mathrm{~nJ}$. To the best of our knowledge, it is the highest pulse energy among Q-switched fiber laser based on BP-SA at $1.5 \mu \mathrm{m}$. The result shows that the PVA-BP-PVA film fabricated by the drop-casting method can provide an ideal SA for high pulse energy generation from fiber lasers, which has a potential application of remote sensing.

\section{Acknowledgment}

This work was supported by the National Natural Science Foundation of China (Grant Nos. 61605108, 61635006, and 61735009); Shanghai Young Oriental Scholar 2016.

Open Access This article is distributed under the terms of the Creative Commons Attribution 4.0 International License (http://creativecommons.org/licenses/by/4.0/), which permits unrestricted use, distribution, and reproduction in any medium, provided you give appropriate credit to the original author(s) and the source, provide a link to the Creative Commons license, and indicate if changes were made.

\section{References}

[1] P. Pérez-Millán, J. L. Cruz, and M. V. Andrés, "Active Q-switched distributed feedback erbium-doped fiber lasers," Applied Physics Letters, 2005, 87(1): 37.

[2] Z. Q. Luo, M. Zhou, J. Weng, G. M. Huang, H. Y. $\mathrm{Xu}$, C. C. Ye, et al., "Graphene-based passively Q-switched dual-wavelength erbium-doped fiber laser," Optics Letters, 2010, 35(21): 3709-3711.

[3] F. N. Xia, H. Wang, D. Xiao, M. Dubey, and A. Ramasubramaniam, "Two-dimensional material nanophotonics," Nature Photonics, 2014, 8(12): 899-907.

[4] Z. P. Sun, D. Popa, T. Hasan, F. Torrisi, E. J. R. Kelleher, J. C. Travers, et al., "A stable, wideband tunable, near transform-limited, graphene-modelocked, ultrafast laser," Nano Research, 2010, 3(9): 653-660.

[5] P. R. Nair, P. Blake, A. N. Grigorenko, K. S. Novoselo, T. J. Booth, T. Stauber, et al., "Fine structure constant defines visual transparency of graphene," Science, 2008, 320(5881): 1308-1308.

[6] Q. H. Wang, K. Kalantar-Zadeh, A. Kis, J. N. Coleman, and M. S. Strano, "Electronics and optoelectronics of two-dimensional transition metal dichalcogenides," Nature Nanotechnology, 2012, 7(11): 699-712.

[7] S. X. Wang, H. H. Yu, H. J. Zhang, A. Z. Wang, M. W. Zhao, Y. X. Chen, et al., "Broadband few-layer MoS2 saturable absorbers," Advanced Materials, 2014, 26(21): 3538-3544.

[8] C. J. Zhao, H. Zhang, X. Qi, Y. Chen, Z. T. Wang, S. C. Wen, et al., "Ultra-short pulse generation by a topological insulator based saturable absorber," Applied Physics Letters, 2012, 101(21): 118.

[9] J. F. Li, H. Y. Luo, B. Zhai, R. G. Lu, Z. N. Guo, H. Zhang, et al., "Black phosphorus: a two-dimension saturable absorption material for mid-infrared Q-switched and modelocked fiber lasers," Scientific Reports, 2016, 6: 30361.

[10] L. K. Li, Y. J. Yu, G. J. Ye, Q. Q. Ge, X. D. Ou, H. $\mathrm{Wu}$, et al., "Black phosphorus field-effect transistors," Nature Nanotechnology, 2014, 9(5): 372-377.

[11] J. Sotor, G. Sobon, W. Macherzynski, P. Paletko, and K. M. Abramski, "Black phosphorus saturable absorber for ultrashort pulse generation," Applied Physics Letters, 2015, 107(5): 440-449.

[12] K. P. Wang, B. M. Szydlowska, G. Z. Wang, X. Y. Zhang, J. J.Wang, J. J. Magan, et al., "Ultrafast 
nonlinear excitation dynamics of black phosphorus nanosheets from visible to mid-infrared," ACS Nano, 2016, 10(7): 6923-6932.

[13] R. J. Suess, M. M. Jadidi, T. E. Murphy, and M. Mittendorff, "Carrier dynamics and transient photobleaching in thin layers of black phosphorus," Applied Physics Letters, 2015, 107(8): 081103.

[14] S. F. Zhang, Y. X. Li, X. Y. Zhang, N. N. Dong, K. P. Wang, D. Hanlon, et al., "Slow and fast absorption saturation of black phosphorus: experiment and modelling," Nanoscale, 2016, 8(39): 17374-17382.

[15] S. F. Zhang, X. Y. Zhang, H. Wang, B. H. Chen, K. $\mathrm{Wu}, \mathrm{K}$. P. Wang, et al., "Size-dependent saturable absorption and mode-locking of dispersed black phosphorus nanosheets," Optical Materials Express, 2016, 6(10): 3159-3168.

[16] H. Ahmad, M. A. M. Salim, K. Thambiratham, S. F. Norizan, and S. W. Harun, "A black phosphorus-based tunable Q-switched ytterbium fiber laser," Laser Physics Letters, 2016, 13(9): 7.

[17] X. C. Su, Y. R. Wang, B. T. Zhang, R. W. Zhao, K. J. Yang, J. L. He, et al., "Femtosecond solid-state laser based on a few-layered black phosphorus saturable absorber," Optics Letters, 2016, 41(9): 1945-1948.

[18] Y. Chen, G. B. Jiang, S. Q. Chen, Z. N. Guo, X. F. $\mathrm{Yu}$, C. J. Zhao, et al., "Mechanically exfoliated black phosphorus as a new saturable absorber for both Q-switching and mode-locking laser operation," Optics Express, 2015, 23(10): $12823-12833$.

[19] H. Yu, X. Zheng, K. Yin, X. G. Cheng, T. Jiang, "Nanosecond passively Q-switched thulium/holmium-doped fiber laser based on black phosphorus nanoplatelets," Optical Materials Express, 2016, 6(2): 603-609.

[20] J. Sotor, G. Sobon, M. Kowalczyk, W. Macherzynski, P. Paletko, and K. M. Abramski, "Ultrafast thulium-doped fiber laser mode locked with black phosphorus," Optics Letters, 2015, 40(16): 3885-3888.

[21] M. Q. Fan, T. Li, S. Z. Zhao, G. Q. Li, X. Gao, K. J. Yang, et al., "Multilayer black phosphorus as saturable absorber for an Er:Lu2O3 laser at similar to $3 \mu \mathrm{m}$," Photonics Research, 2016, 4(5): 181-186.

[22] S. C. Liu, Y. N. Zhang, L. Li, Y. G. Wang, R. D. Lv, X. Wang, et al., "Er-doped Q-switched fiber laser with a black phosphorus/polymethyl methacrylate saturable absorber," Applied Optics, 2018, 57(6): 1292-1295.
[23] F. Bonaccorso and Z. P. Sun, "Solution processing of graphene, topological insulators and other $2 \mathrm{~d}$ crystals for ultrafast photonics," Optical Materials Express, 2013, 4(1): 63-78.

[24] J. Y. Liu, F. Y. Zhao, H. S. Wang, W. Zhang, X. H. $\mathrm{Hu}, \mathrm{X} . \mathrm{H}$. Li, et al., "Generation of dark solitons in erbium-doped fiber laser based on black phosphorus nanoparticles," Optical Materials, 2019, 89: 100-105.

[25] Z. N. Guo, H. Zhang, S. B. Lu, Z. T. Wang, S. Y. Tang, J. D. Shao, et al., "From black phosphorus to phosphorene: basic solvent exfoliation, evolution of Raman scattering, and applications to ultrafast photonics," Advanced Functional Materials, 2015, 25(45): 6996-7002.

[26] Q. L. Feng, H. Y. Liu, M. J. Zhu, J. Shang, D. Liu, X. Q. Cui, et al., "Electrostatic functionalization and passivation of water-exfoliated few-layer black phosphorus by poly dimethyldiallyl ammonium chloride and its ultrafast laser application," $A C S$ Applied Materials \& Interfaces, 2018, 10: 9679-9687.

[27] A. Castellanos-Gomez, L. Vicarelli, E. Prada, J. O. Island, K. L. Narasimha-Acharya, S. I. Blanter, D. J. Groenendiijk, et al., "Isolation and characterization of few-layer black phosphorus," $2 d$ Materials, 2014, 1(2): 025001.

[28] Y. Zhang, X. H. Li, A. Qyyum, T. C. Feng, P. L. Guo, J. Jiang, et al., "PbS nanoparticles for ultrashort pulse generation in optical communication region," Particle \& Particle Systems Characterization, 2018, 35: 1800341.

[29] X. H. Li, X. C. Yu, Z. P. Sun, Z. Y. Yan, B. Sun, Y. B. Cheng, et al., "High-power graphene mode-locked $\mathrm{Tm} / \mathrm{Ho}$ co-doped fiber laser with evanescent field interaction," Scientific Reports, 2015, 5: 16624.

[30] H. R. Mu, S. H. Lin, Z. X. Wang, S. Xiao, P. F. Li, Y. Chen, et al., "Black phosphorus-polymer composites for pulsed lasers," Advanced Optical Materials, 2015, 3(10): 1447-1453.

[31] D. Li, A. Esau Del Rio Castillo, H. Jussila, G. J. Ye, Z. Y. Ren, J. T. Bai, et al., "Black phosphorus polycarbonate polymer composite for pulsed fibre lasers," Applied Materials Today, 2016, 4: 17-23.

[32] D. Li, H. R. Jussila, L. Karvonen, G. J. Ye, H. Lipsanen, X. H. Chen, et al., "Polarization and thickness dependent absorption properties of black phosphorus: new saturable absorber for ultrafast Pulse generation," Scientific Reports, 2015, 5: 15899. 